\title{
Polymer-Silica Hybrids: Evaluation of Grafted Poly(acrylonitrile) as Organic Phase for High-Performance Liquid Chromatography
}

\author{
Hirotaka IHARA*†, Shinya OKAZAKI*, Kanako OHMORI*, Shinobu UeMURA*, \\ Chuichi HiRAYAMA* and Shoji NAGAOKA** \\ *Department of Applied Chemistry \& Biochemistry, Faculty of Engineering, Kumamoto University, \\ Kumamoto 860-8555, Japan \\ **Kumamoto Industrial Research Institute, Kumamoto 860-0901, Japan
}

\begin{abstract}
Poly(acrylonitrile) with a terminal reactive group at one side of the main chain was prepared by telomerization of acrylonitrile with 3-mercaptopropyltrimethoxysilane and then immobilized onto porous silica. HPLC using the packed column $\left(\right.$ Sil- $\left.\mathrm{AN}_{n}\right)$ and an aqueous solution as a mobile phase showed several unique retention behaviors for hydrocarbons: (1) the retention factor for alkylbenzenes was almost independent of the alkyl length although simply cyanopropylated silica (Sil$\mathrm{CN}$ ) showed the increase of the retention time with increase of the alkyl length. (2) Sil- $\mathrm{AN}_{n}$ showed remarkably better selectivity for $\pi$-electron containing hydrocarbons compared with Sil-CN and hydrophobized silica. In this paper, such specificity of $\mathrm{Sil}_{-} \mathrm{AN}_{n}$ is discussed in relation to $\pi$-electron recognition ability of poly(acrylonitrile) moiety.
\end{abstract}

Keywords Liquid chromatography, silica, polymer-grafting, $\pi-\pi$ interaction

Lipid membranes work as the most important medium for generation of various biofunctions in which precise separation and permeation of chemical substances are included. Therefore, immobilization of lipid membrane functions onto carriers promises to increase possible applications. Especially in the field of separation chemistry, biomimetical precise separation would be reproduced. Direct immobilization of lipids ${ }^{1}$ and polymerization of lipids ${ }^{2-7}$ are known as useful methods to stabilize lipids and lipid membranes. However, it should be noted that these techniques are generally accompanied by disappearance of lipid membrane functions because of suppressing the lateral diffusion of lipids, which is the most important property. To overcome this dilemma, we have developed a method to graft the comb-shaped polymer $\left(\mathrm{ODA}_{n}\right.$, in Fig. 1) onto

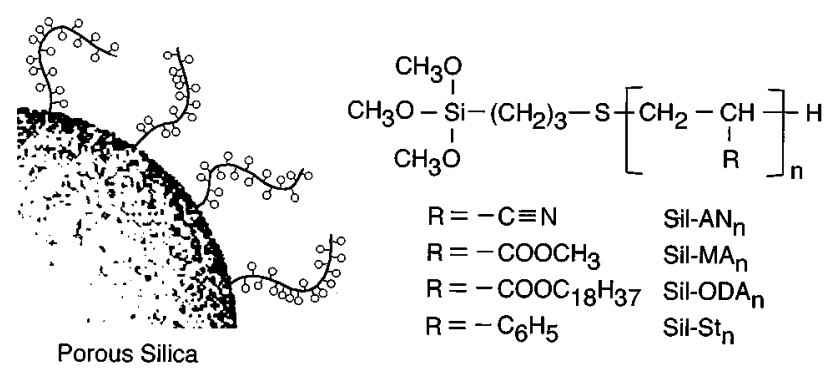

Fig. 1 Schematic illustration of linear polymer-grafted silica. silica through a terminal reactive group. ${ }^{8}$ The polymer does not produce lipid bilayer membrane structures in water but forms a highly-oriented structure and shows a phase transition on silica like aqueous lipid membranes even after immobilization. We have also reported that the silica-supported $\mathrm{ODA}_{n}$ shows unique HPLC separations recognizing molecular planarity ${ }^{8-10}$, -bulkiness ${ }^{11}$ and -length ${ }^{10,11}$ as well as -hydrophobicity.

One of the advantages in this polymer-grafting method is in the fact that the immobilized polymers are free from perturbation of supporting materials and thus show individual functions, as the polymer can be chemically combined to silica at one side of the terminal groups. This advantage assures applicability for various polymers. ${ }^{12-14}$ In this paper, we report grafting of poly(acrylonitrile), $\mathrm{AN}_{n}$ onto silica and its HPLC performance for the first time. $\mathrm{AN}_{n}$ provides small hydrophobicity and a strong $\pi$-electron effect, which allow unique separation behaviors in HPLC.

\section{Experimental}

\section{Materials}

Synthetic procedure of poly(acrylonitrile), $\mathrm{AN}_{n}$, where $n$ is the average polymerization degree, is as follows: acrylonitrile and 3-mercaptopropyltrimethoxysilane (10: 1 in the molar ratio) were dissolved in ethanol. Azobisisobutyronitrile (0.1 wt $\%$ for the monomer) was added to the solution at $60^{\circ} \mathrm{C}$. The mix- 
ture was stirred for $72 \mathrm{~h}$ at $60^{\circ} \mathrm{C}$ under $\mathrm{N}_{2}$ gas atmosphere. The white precipitates obtained were gathered by concentration and filtration, washed successively with ethanol and dried in vacuo. The structure and polymerization degree of the polymer were determined by NMR spectroscopy: ${ }^{1} \mathrm{H}-\mathrm{NMR}$ chemical shifts of $\mathrm{AN}_{n}$ in $d_{6}$-dimethylsulfoxide were as follows: $\delta=2.05$ ppm $\left(\mathrm{C}_{2} \mathrm{CHCN}\right), \delta=3.15 \mathrm{ppm}\left(\mathrm{CH}_{2} \mathrm{C} \underline{\mathrm{H}}-\mathrm{CN}\right)$ and $\delta=3.55 \mathrm{ppm}\left(\mathrm{SiOC}_{3}\right)$.

$\mathrm{AN}_{21}$ was readily introduced onto porous silica by mixing in dimethylformamide at $80^{\circ} \mathrm{C}$. YMC 120 -S5 (diameter $5 \mu \mathrm{m}$, pore size $120 \AA, 300 \mathrm{~m}^{2} \mathrm{~g}^{-1}$ ) were used as porous silica. Successive washing of the resulting gels with dimethylformamide and ether followed. The amount of $\mathrm{AN}_{21}$ introduced was determined by elemental analysis.

Wakopak (Wakosil 5CN, $4.6 \mathrm{~mm}$ i.d. $\times 250 \mathrm{~mm}$ ) was used as a $\mathrm{CN}$-containing silica (Sil-CN). ODS was used as a simply-hydrophobized silica (Inertsil ODS, $4.6 \mathrm{~mm}$ i.d. $\times 300 \mathrm{~mm}$ ).

\section{Measurements}

Silica-supported $\mathrm{AN}_{21}\left(\mathrm{Sil}-\mathrm{AN}_{21}\right)$ was packed into a stainless-steel column $(4.6 \mathrm{~mm}$ i.d. $\times 250 \mathrm{~mm})$ using a hexanol-chloroform (1:1) mixture. The liquid chromatographic property was examined using methanol or methanol-water as mobile phases. The chromatograph included a JASCO 880 PU pump, and a Waters 996 photodiode array detector. Five microliters of the sample dissolved in methanol was injected through a Rheodyne Model 7125 injector. Chromatography was carried out at a flow-rate of $1.0 \mathrm{ml} \mathrm{min}{ }^{-1}$. The retention factor $\left(k^{\prime}\right)$ was determined by $\left(t_{\mathrm{e}}-t_{\mathrm{o}}\right) / t_{\mathrm{o}}$, where $t_{\mathrm{e}}$ and $t_{\mathrm{o}}$ are retention time of samples and methanol, respectively. The separation factor $(\alpha)$ was given by the ratio of retention factors.

1-Octanol-water partition coefficient $(\log P)^{15}$ was determined by the retention factor with octadecylated silica, ODS (Inertsil ODS, $4.6 \mathrm{~mm}$ i.d. $\times 300 \mathrm{~mm}$, GL Science Co., Ltd.): $\log P=3.262+4.208 \log k^{\prime}$.

\section{Calculation}

The structures of $\mathrm{AN}_{21}$ was estimated using SonyTektronix CAChe-mechanics (the optimization with MM2 parameters).

\section{Results and Discussion}

\section{Preparation of silica-supported poly(acrylonitrile)}

Poly(acrylonitrile) with a terminal trimethoxysilyl group $\left(\mathrm{AN}_{n}\right.$, where $n$ is the average polymerization degree) was prepared by telomerization of acrylonitrile and 3-mercaptopropyltrimethoxysilane. The polymerization degree $(n)$ of $\mathrm{AN}_{n}$ was determined to be 21 by ${ }^{1} \mathrm{H}-\mathrm{NMR}$ spectroscopy (in $d_{6}$-dimethylsulfoxide, $\delta=3.15 \mathrm{ppm}\left(\mathrm{CH}_{2} \mathrm{C} \underline{\mathrm{H}}-\mathrm{CN}\right)$ and $\delta=3.55 \mathrm{ppm}\left(\mathrm{SiOC}_{3}\right)$.

The $\mathrm{AN}_{21}$ was readily immobilized on porous silica by mixing at $80^{\circ} \mathrm{C}$ in dimethylformamide. Successive washing of resulting silica with dimethylformamide showed no change in weight. IR spectroscopy showed the specific absorption due to $\mathrm{CN}$ groups $\left(v_{\mathrm{CN}}, 2273\right.$ $\mathrm{cm}^{-1}$ ). The elemental analysis showed that $13.8 \mathrm{wt} \%$ (C, $8.88 \mathrm{wt} \% ; \mathrm{N}, 3.02 \mathrm{wt} \%$ ) of $\mathrm{AN}_{21}$ was introduced on the silica. When $\mathrm{AN}_{21}$ is represented by a rod-like molecules with a maximum surface area of $411 \AA^{2}(52 \times 7.9$ $\AA)^{16}, 1 \mathrm{~g}$ of silica surface with $300 \mathrm{~m}^{2} \mathrm{~g}^{-1}$ can be covered with $0.135 \mathrm{~g}$ of $\mathrm{AN}_{21}$. This coverage corresponds to the resulting immobilized amount (13.8 wt\%).

\section{Retention behavior for polyaromatic hydrocarbons}

Figure 2a shows a typical chromatogram of a mixture of benzene, naphthalene, anthracene, pyrene and triphenylene with a Sil- $\mathrm{AN}_{21}$ column and a methanol-water
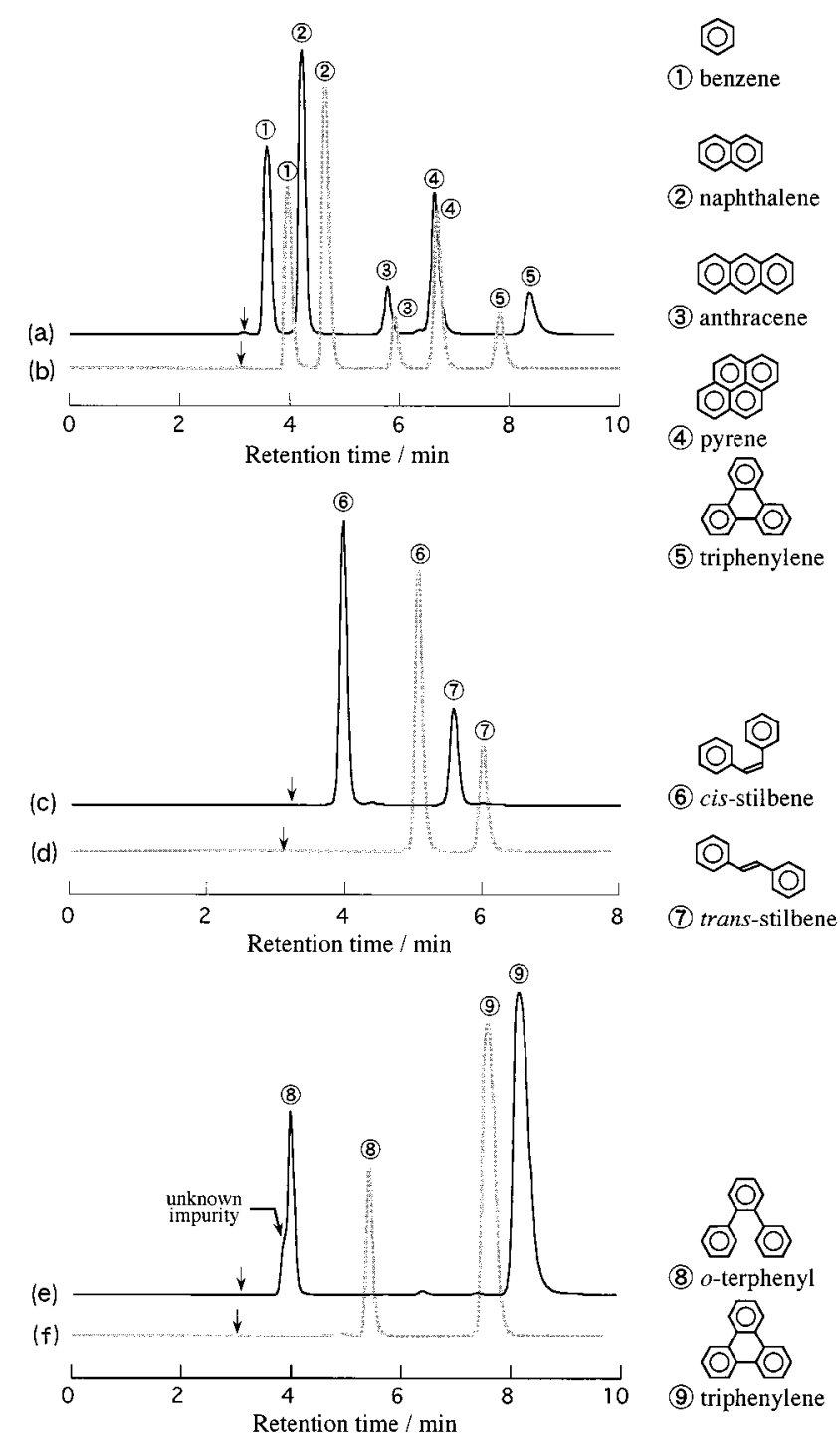

Fig. 2 Typical chromatograms with $\operatorname{Sil}_{-} \mathrm{AN}_{21}$ (a, c, and e) and Sil-CN (b, d and f) columns at $25^{\circ} \mathrm{C}$. Mobile phase, methanolwater $(7: 3)$. The small arrows indicate $t_{0}$. The unknown impurity in a chromatogram (e) provided a UV spectrum with $\lambda_{\max } \mathrm{s}$ of $224 \mathrm{~nm}$ and $276 \mathrm{~nm}$ (small) although $o$-terphenyl has a $\lambda_{\max }$ at $233 \mathrm{~nm}$. 
mixture as a mobile phase. The elution order (Fig. 3a) is the same of typical RPLC mode. Specific behavior of Sil- $\mathrm{AN}_{21}$ appeared for alkylbenzenes. As shown in Fig. 3a, the retention factor is independent of the alkyl length of samples. No similar specificity was observed in simply-cyanopropylated silica (Sil-CN, Wakosil $5 \mathrm{CN})$. These results indicate that Sil- $\mathrm{AN}_{21}$ does not recognize molecular hydrophobicity (the number of methylenes) although it is sensitive to molecular aromaticity.

\section{Separation of geometrical isomers of stilbenes}

As shown in Fig. 2c, Sil-AN ${ }_{21}$ provides a much better separation factor $(\alpha=2.25)$ for the geometrical isomers of stilbenes than Sil-CN ( $\alpha=1.52)$, although an extremely small separation factor $(\alpha=1.1-1.2)$ was observed in simply-hydrophobized silica (ODS, Inertsil ODS). To understand this difference, separation factors with various mobile phases were examined. As a result, the separation factor increased with increase of the water-content when methanol or ethanol were used as mobile phases: $\alpha=1.4,1.8$ and 3.2 for $0 / 100,20 / 80$ and $50 / 50$ $\mathrm{v} / \mathrm{v} \%$ in a water-methanol mixture. On the contrary, the separation factor was small and almost constant
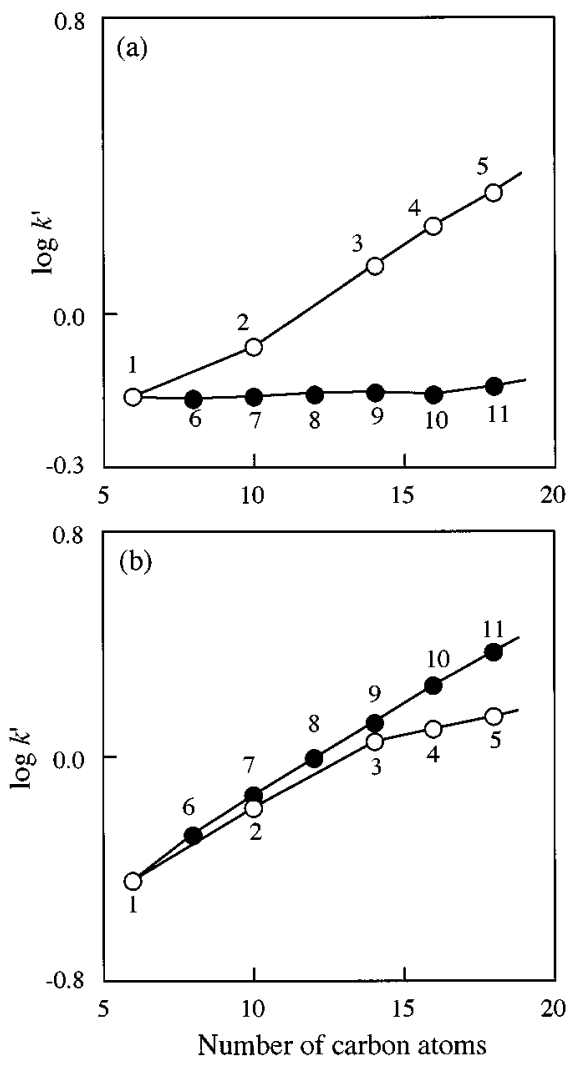

Fig. 3 Relationships between $\log k^{\prime}$ and the number of carbon atoms of elutes with Sil-AN $\mathrm{AN}_{21}$ (a) and Sil-CN (b) columns at $25^{\circ} \mathrm{C}$. Mobile phase, methanol-water (7:3). Elutes: 1, benzene; 2 , naphthalene; 3 , anthracene; 4, pyrene; 5 , triphenylene; 6 - 11, ethyl-, butyl-, hexyl-, octyl-, decyl- and dodecylbenzenes.
( $\alpha=1.1-1.2$ ) independent of the water-content when acetonitrile $\left(\mathrm{CH}_{3} \mathrm{CN}\right)$ was used as mobile phases instead of methanol or ethanol. Similar observations were obtained in the separation of a mixture of triphenylene and $o$-terphenyl. These samples have the same numbers of carbon atoms but the big differences in molecular planarity and aromaticity. As shown in Figs. $2 \mathrm{e}$ and $2 \mathrm{f}, \mathrm{Sil}_{-} \mathrm{AN}_{21}$ provided a much better separation factor $(\alpha=6.05)$ with methanol-water than Sil-CN $(\alpha=2.33)$ and ODS $(\alpha=1.60)$. These results strongly suggest that grafted $\mathrm{CN}$ groups work as essential interaction groups for generation of selective retention. The recognition mechanism will be discussed in the next sections.

\section{Evaluation of $\mathrm{CN}-\pi$ interaction}

$\mathrm{CN}-\pi$ interaction between $\mathrm{Sil}-\mathrm{AN}_{21}$ and $\pi$-electron containing-compounds is expected as a driving force for retention selectivity. In order to clarify the contribution of $\mathrm{CN}$ groups for the selectivity, we investigated the retention behavior for various cyclic hydrocarbons with $\pi$-electrons. Figure 4 a shows the relationship between $\log k^{\prime}$ and $\log P^{15}$ as a parameter of molecular hydrophobicity. If Sil- $\mathrm{AN}_{21}$ has hydrophobicity recognition ability similar to ODS, a linear correlation must be observed. However, the plot rather indicates molecular size (the number of cyclic rings) recognition. Therefore, the $\log k^{\prime}-\log P$ plot provided a very small correlation coefficient, $r=0.57$.

On the basis of these findings, in this paper, we consider a new parameter $\boldsymbol{f}$ to evaluate the contribution of $\pi-\pi(\mathrm{CN}-\pi)$ interaction although Schabron et al. propose an $\boldsymbol{F}$ parameter considering the contribution of double bonds of elutes, which is given by the following equation: $\boldsymbol{F}=$ the number of double bonds+the number of primary and secondary carbon atoms-0.5 for a nonaromatic ring. ${ }^{17}$ This parameter $\boldsymbol{F}$ has been often used to characterize retention behavior for $\pi$-electroncontaining compounds. ${ }^{10,18}$ However, the $\log \boldsymbol{k}^{\prime}-\boldsymbol{F}$ plot maintained still a very small correlation coefficient ( $r=0.62$ for Sil-AN $\mathrm{AN}_{21}$ in Fig. 4b). On the other hand, our $f$ parameter is presented as the following equation:

\section{$\boldsymbol{f}=f_{\mathrm{H}}$ for the number of $\mathrm{sp}^{3}$ carbon atoms $+f_{\mathrm{P}}$ for the number of $\mathrm{sp}^{2}$ carbon atoms $\beta=f_{\mathrm{P}} / f_{\mathrm{H}}$}

Here the parameter $\boldsymbol{f}$ does not characterize the absolute chemical potential of a solute but is used to estimate what degree interacts with stationary phase, which an $\boldsymbol{F}$ parameter is the descriptor showing only the solute property. Therefore, a parameter of $\beta$ (the ratio of $f_{\mathrm{P}}$ and $f_{\mathrm{H}}$ ) in $f$ provides the most significant information to understand the retention mechanism. If the stationary phase has no specific interaction such as $\pi-\pi$ interaction with $\mathrm{sp}^{2}$ carbon atoms of samples, $\beta$ value will become below 1 , because $\mathrm{sp}^{2}$ carbon atoms behave as more polar moiety than $\mathrm{sp}^{3}$ carbon atoms in RPLC mode.

To confirm applicability of these new parameters to 

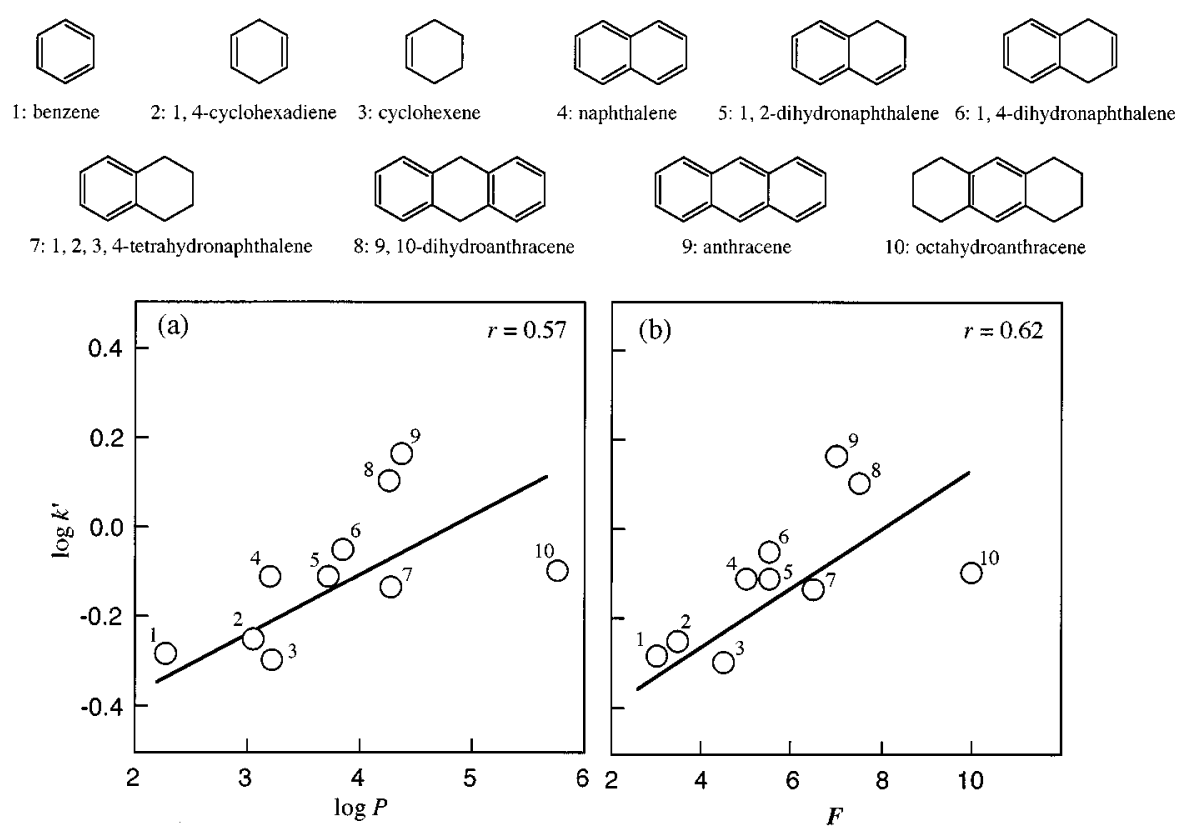

Fig. 4 Plots of $\log k^{\prime}$ against $\log P$ and $\boldsymbol{F}$ parameters with Sil- $\mathrm{AN}_{21}$ column at $25^{\circ} \mathrm{C}$. Mobile phase, methanol-water (7:3).
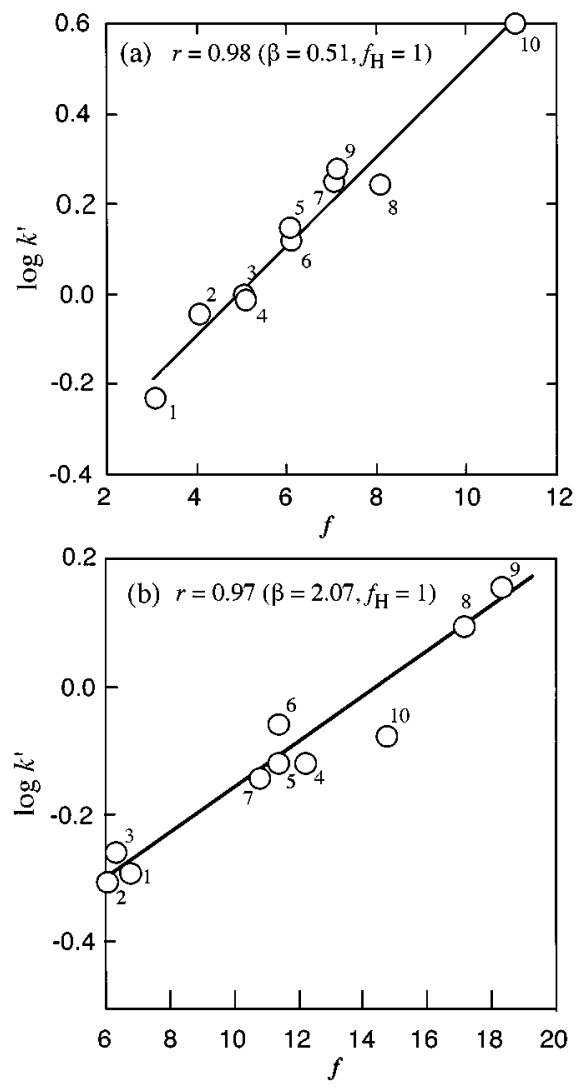

Fig. 5 Plots of $\log k^{\prime}$ against $f$ parameters with ODS (a) and Sil- $\mathrm{AN}_{21}$ (b) column at $25^{\circ} \mathrm{C}$. Mobile phase, methanol-water (9:1 in ODS, 7:3 in Sil-AN ${ }_{21}$ ). The samples listed in Fig. 4 were used. our chromatographic results, the relationship between $\log k^{\prime}$ and $f$ was examined with ODS. As shown in Fig. $5 \mathrm{a}$, the plot provided a good linearity with $r=0.98$ as a maximum correlation factor, when $\beta$ was given as 0.51 . Since octadecyl groups in ODS have almost no $\pi$-electron affinity, this small $\beta$ value $(0.51)$ indicates that the $\mathrm{sp}^{2}$ carbon atoms do not work as $\pi-\pi$ interaction source but behave as hydrophobic source with approximately half of hydrophobicity for the $\mathrm{sp}^{3}$ carbon atoms in this liquid chromatographic condition. Figure $5 \mathrm{~b}$ shows the $\log k^{\prime}-f$ plot for Sil- $\mathrm{AN}_{21}$. The best linearity was obtained when $\beta$ was calculated as 2.07. The correlation coefficient $(r=0.97)$ is much better than those in the plots of the $\log k^{\prime}-\log P(r=0.57)$ and $\log k^{\prime}-\boldsymbol{F}$ $(r=0.62)$. Here, it should be noted that the $\beta$ value is much higher than that in ODS. This strongly indicates that $\mathrm{AN}_{21}$ interacts efficiently with the $\mathrm{sp}^{2}$ carbon atoms.

\section{Application of $f$ parameter in hexane}

$\mathrm{CN}$ groups of $\mathrm{AN}_{21}$ are too hydrophilic to work as hydrophobic organic phase. However, small hydrophobicity can be provided by the main chain of $\mathrm{AN}_{21}$. If a non-polar solvent such as hexane is used as a mobile phase, hydrophobic retention must be negligible in liquid chromatography and thus the molecular recognition mechanism must become simpler than when a polar solvent is used. In the case of ODS, almost no retention was observed in hexane for all the samples which were listed in Fig. 3. This is due to the fact that ODS recognizes only molecular hydrophobicity. On the contrary, Sil- $\mathrm{AN}_{21}$ provided significant retention for these samples. Figure 6 shows the plot of $\log k^{\prime} v s . f$ in hex- 


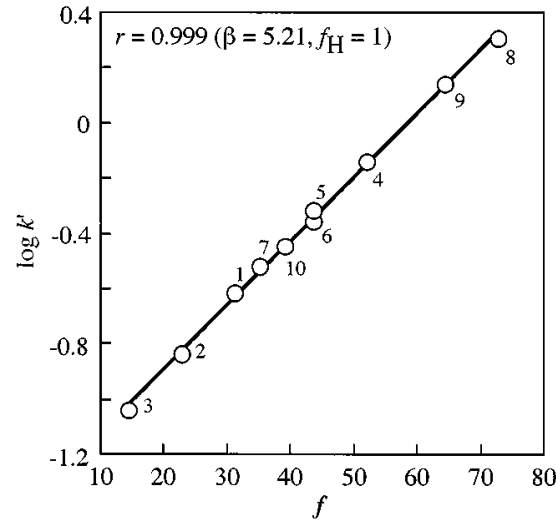

Fig. 6 Plot of $\log k^{\prime} v s$. $f$ plot with Sil- $\mathrm{AN}_{21}$ column at $25^{\circ} \mathrm{C}$. Mobile phase, hexane. The samples listed in Fig. 4 were used.

ane. When $\beta$ is characterized by 5.21 , the plot provided the best linearity $(r=0.999)$. This extremely large value indicates that the interaction is absolutely derived from the $\mathrm{sp}^{2}$ carbon atoms in hexane.

\section{Application of $\boldsymbol{f}$ parameter to various modified silicas}

We emphasize that an $\boldsymbol{f}$ parameter is applicable to various modified silicas. Table 1 shows the correlation coefficient in the plots of $\log k^{\prime}-\log P,-\boldsymbol{F}$ and $\boldsymbol{f}$ with Sil-MA ${ }_{21}$ and Sil-St ${ }_{16}$ which poly(methyl acrylate) and poly(styrene) are grafted onto silicas. An $f$ parameter provided good correlation coefficients for all the silicas by adjusting $f_{\mathrm{P}}$ and $f_{\mathrm{H}}$ values, but the $\beta\left(f_{\mathrm{P}} / f_{\mathrm{H}}\right)$ value is the largest in Sil- $\mathrm{AN}_{21}(2.07)$ and reduces in the order of Sil-MA $\mathrm{MA}_{21}{ }^{14}$ (1.18), Sil-St ${ }_{16}{ }^{12}$ (0.75) and ODS (0.51). This order will be explained as follows: octadecyl groups in ODS are the most hydrophobic and have no $\pi$-electron. Therefore, ODS shows the smallest $\beta$ value (0.51). On the other hand, Sil- $\mathrm{AN}_{21}$, Sil- $\mathrm{MA}_{21}$ and Sil$\mathrm{St}_{16}$ have $\pi$-electrons as $\pi-\pi$ interaction sources. This explains that the $\beta$ values are higher than that of ODS. In addition, the hydrophobicity of $\mathrm{CN}, \mathrm{COOCH}_{3}$ and phenyl groups as the side chains differ from each other, although the main chain is the completely same in these chemical structures. Therefore, it is reasonable that Sil$\mathrm{AN}_{21}$ with the most hydrophilic $\mathrm{CN}$ group provides the largest $\beta$ value (2.07) and $\mathrm{Sil}_{-\mathrm{St}_{16}}$ with the most hydrophobic phenyl group provides the smallest $\beta$ value (0.76): in Sil-St ${ }_{16}$ the $\pi$-electron effect is comparably diluted by the large hydrophobicity.

\section{Difference between Sil-CN and Sil-AN $N_{21}$}

Selectivity derived from $\mathrm{CN}-\pi$ interaction must appeared even in simply-cyanopropylated silica (Sil$\mathrm{CN}$ ). As shown in Fig. 2, Sil-CN provides good selectivity for polyaromatic hydrocarbons and their geometrical isomers. These separation factors are larger than in ODS but smaller than in Sil-AN ${ }_{21}$. The $\log k^{\prime}-f$ plot in Sil-CN showed a good linearity $(r=0.98)$ when $\beta$ is 1.02. This $\beta$ value is also larger than that of ODS $(0.51)$ but significantly smaller than that of Sil- $\mathrm{AN}_{21}$ (2.07). This is probably attributable to a hydrophobic effect derived from propyl spacers in Sil-CN, while $\mathrm{CN}-\pi$ interaction is included in the retention mechanism. The hydrophobicity reduces the selectivity for $\pi$ electron-containing substances.

In conclusion, silica-poly(acrylonitrile) hybrids (Sil$\mathrm{AN}_{21}$ ) have been prepared for HPLC use. This new packing material showed some unique properties: (1) Sil-AN ${ }_{21}$ showed longer retention and better selectivity for polyaromatic hydrocarbons than simplycyanopropylated silica. (2) Sil- $\mathrm{AN}_{21}$ recognizes molecular aromaticity but not hydrophobicity in a polar solvent as a mobile phase. (3) The good selectivity appears in geometrical isomers of stilbenes. It is estimated that these unique properties are derived from a $\pi$-electron effect of $\mathrm{CN}$ groups. These findings are supported by both establishments of a hybridization method of polymers with silica and proposal of a new parameter $\boldsymbol{f}$ for evaluation of $\pi-\pi$ interaction.

\section{References}

1. C. Pidgeon and U. V. Venkataram, Anal. Biochem., 176, 36 (1989).

2. H. Ihara, M. Takafuji, C. Hirayama and D. F. O'Brien, Langmuir, 8, 1548 (1992).

3. D. F. O'Brien and R. Ramasuwami, "Encyclopedia of Polymer Science and Engineering, 2nd ed"., J. Wiley \& Sons, New York, 17, 108 (1989).

Table 1 Comparison of correlation coefficient $(r)$ in the plots of $\log k^{\prime}-\log P, \log k^{\prime}-\boldsymbol{F}$ and $\log k^{\prime}-\boldsymbol{f}$

\begin{tabular}{llcccc}
\hline \multirow{2}{*}{ Organic phase } & \multicolumn{4}{c}{ Correlation coefficient $(r)$} \\
\cline { 3 - 5 } & & $\log P$ & $\boldsymbol{F}$ & $\boldsymbol{f}$ & $\beta$ \\
\hline Sil-AN $_{21}$ & poly(acrylonitrile) & 0.57 & 0.62 & 0.97 & 2.07 \\
Sil-MA $_{21}$ & poly(methyl acrylate) & 0.78 & 0.83 & 0.99 & 1.18 \\
Sil-St $_{16}$ & poly(styrene) & 0.91 & 0.96 & 0.99 & 0.79 \\
ODS $^{\text {a }}$ & octadecyl & - & 0.98 & 0.98 & 0.51 \\
\hline
\end{tabular}

Mobile phase, methanol:water $=7: 3$ except for ODS (methanol: water $=9: 1$ ) ${ }^{\mathrm{a}}$; temperature, $25^{\circ} \mathrm{C}$; samples given in Fig. 4 were used. 
4. H. Ringsdorf, B. Schlarb and J. Venzmer, J. Angew. Chem., Int. Ed. Engl., 27, 113 (1988).

5. S. L. Regen, In "Liposomes: From Biophysics to Therapeutics", ed. M. J. Ostro, p. 73, Mercel Dekker, New York 1987.

6. J. H. Fendler, Science [Washington, D. C.], 223, 888 (1984).

7. T. Kunitake, N. Nakashima, K. Takarabe, M. Nagai, A. Tsuge and H. Yanagi, J. Am. Chem. Soc., 103, 5945 (1981).

8. C. Hirayama, H. Ihara and T. Mukai, Macromol., 25, 6357 (1992).

9. T. Fukumoto, H. Ihara, S. Sakaki, H. Shosenji and C. Hirayama, J. Chromatogr., 672, 237 (1994).

10. H. Ihara, H. Tanaka, S. Nagaoka, S. Sakaki and C. Hirayama, J. Liq. Chromatogr., 19, 2967 (1996).

11. H. Ihara, T. Fukumoto and C. Hirayama, Anal. Sci., 9, 711 (1993).

12. H. Ihara, N. Nakamura, S. Nagaoka and C. Hirayama, Anal. Sci., 11, 739 (1995)
13. C. Hirayama, H. Ihara, S. Nagaoka and T. Wada, Polym. J., 26, 499 (1994)

14. H. Ihara, H. Tanaka, M. Shibata, S. Sakaki and C. Hirayama, Chem. Lett., 1997, 113.

15. F. Rekker and H. M. de Kort, Eur. J. Med. Chem., 479, 14 (1979).

16. Calculation was applied for syndiotactic poly(acrylonitrile) with the degree of polymerization, 21 because hetrotacticity produced innumerable conformations. Calculation was continued until the energy change was less than $0.001 \mathrm{kcal}$ $\mathrm{mol}^{-1}$.

17. J. F. Schabron, R. J. Hurtubise and H. F. Silver, Anal. Chem., 49, 2253 (1977).

18. K. Jinno, Y. Saito, R. Malhan Chopra, J. J. Pesek, J. C. Fetzer and W. R. Biggs, J. Chromatogr., 459, 557 (1991).

(Received August 21, 1997)

(Accepted November 21, 1997) 